\title{
Water evaporation particularities in the process of forest fire extinguishing
}

\author{
Pavel A. Strizhak, Roman S. Volkov ${ }^{a}$, Olga V. Vysokomornaya and Ivan S. Voytkov \\ National Research Tomsk Polytechnic University, 634050 Tomsk, Russia
}

\begin{abstract}
Numerical simulation of water massif motion through the high temperature gases corresponding to the typical conditions of forest fires was carried out. Maximal values of part by volume of liquid evaporating from water massif under its motion through the flaming burning area were determined when solving the heat and mass transfer problem under the conditions of endothermic phase transformations. Influence of liquid phase transition heat on the heat and mass transfer conditions on the track of water massif was determined. The expediency of polydisperse interspaced in time and space atomization of water massifs under the large-scale (especially, forest fires) fire extinguishing was proved.
\end{abstract}

\section{Introduction}

One of the most widespread methods of influence on forest fires for the purpose of its localization and speed limitation of flame spread is the introduction of sufficiently large (up to several tons) water weights in the burning area. Therefore, the extinguishing liquid is dropped sufficiently compactly (without preliminary atomization) in the form of "water slug", as a rule, in the flame massif. Until the present time, the experimental data about the influence principles of moving through the flame "water slug" on the temperature decrease process in the burning area in the territory being worked using the aviation. Also, any information about how the water is efficiently used under considered conditions of its introduction in the flame massif is lacking. The usage completeness evaluation of phase transformation in motion of "water slug" through the flame massif of certain height is appropriate for further development of forest fire extinguishing technologies.

The solution of this problem with full statement taking into account the influence of all significant factors and processes is yet, obviously, impossible. The numerical solution results of problems about the single water droplet motion [1] and the group of such droplets disposed in a definite way relative to each other $[2,3]$ through the high temperature combustion products of different substances show the necessity of very time-consuming computations for the simulation of heat and mass transfer processes and phase transformations even with using of relatively simple models [1-3].

The purpose of this article is a utilization efficiency evaluation of water evaporation completeness in motion of "water slug" through the massif of high temperature combustion products of forest fuel materials based on the simplified model of heat transfer.

\footnotetext{
${ }^{a}$ Corresponding author: romanvolkov@tpu.ru
}

This is an Open Access article distributed under the terms of the Creative Commons Attribution License 4.0, which permits unrestricted use, distribution, and reproduction in any medium, provided the original work is properly cited. 

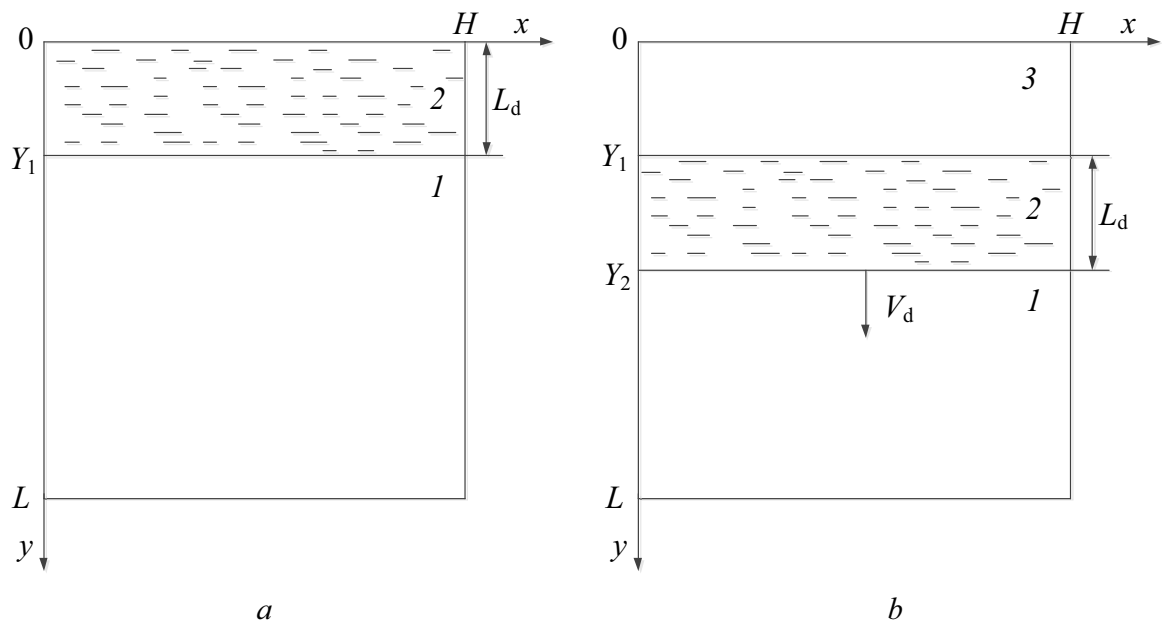

Figure 1. A schematic illustration of problem solution region: 1 - high temperature gases, 2 - "water slug", 3 water vapors.

\section{Problem statement and solution methods}

The basic principle under the problem statement is an evaporation completeness of all liquid in "water slug" under its motion through the high temperature gases. It is expected that the motion time is a characteristic lifetime td of "slug", but the volume evaporation velocity is equal to the water inflow velocity in the actual volume. All liquid captured by elementary volume during the short period of time (Fig. 1) corresponding the "slug" motion speed is fully evaporated (the evaporation front moves through the flame with this velocity).

Such statement despite the certain difficulties in the process of its implementation significantly simplifies the analysis of a complex of the heat and mass transfer processes [1-3] typical of conditions under consideration. Temperature fields and isotherms obtained correspond the variant of full flashing according to results of numerical analysis (idealization of real situation allowing provide an answer about the completeness of water evaporation during the forest fire extinguishing with the application of aviation).

\section{Mathematical model and solution method}

Mathematical model of heat transfer processes at $0<t<t_{\mathrm{d}}$ includes the thermal conduction equations for "water slug - water vapors - flame" system (Fig. 1,b):

$$
\begin{aligned}
& \frac{\partial T_{1}}{\partial t}=a_{1}\left[\frac{\partial^{2} T_{1}}{\partial x^{2}}+\frac{\partial^{2} T_{1}}{\partial y^{2}}\right] \text { at } 0<x<H, Y_{2}<y<L, \\
& \frac{\partial T_{2}}{\partial t}=a_{2}\left[\frac{\partial^{2} T_{2}}{\partial x^{2}}+\frac{\partial^{2} T_{2}}{\partial y^{2}}\right] \text { at } 0<x<H, Y_{1}<y<Y_{2}, \\
& \frac{\partial T_{3}}{\partial t}=a_{3}\left[\frac{\partial^{2} T_{3}}{\partial x^{2}}+\frac{\partial^{2} T_{3}}{\partial y^{2}}\right] \text { at } 0<x<H, 0<y<Y_{1} .
\end{aligned}
$$

$Y_{1}=V_{\mathrm{d}} t, Y_{2}=L_{\mathrm{d}}+V_{\mathrm{d}} t$, where $t-$ time, $\mathrm{s} ; t_{\mathrm{d}}-$ full flashing time (existence) "water slug", $\mathrm{s} ; T-$ temperature, $\mathrm{K} ; a$ - temperature conductivity coefficient, $\mathrm{m}^{2} / \mathrm{s}(a=\lambda /(C \rho)) ; \lambda$ - thermal conductivity 
coefficient, $\mathrm{W} /(\mathrm{m} \cdot \mathrm{K}) ; C$ - coefficient of heat capacity, $\mathrm{J} /(\mathrm{kg} \cdot \mathrm{K}) ; \rho$ - density, $\mathrm{kg} / \mathrm{m}^{3} ; x, y$ - Cartesian ordinates, $\mathrm{m} ; H, L$ - solution region dimensions, $\mathrm{m} ; V_{\mathrm{d}}$ - linear motion speed of "slug", $\mathrm{m} / \mathrm{s} ; L_{\mathrm{d}}-$ characteristic longitudinal dimension of "slug", m; indexes: 1 - high temperature gases, 2 - "water slug", 3 - water vapors.

Initial $(t=0)$ conditions (Fig. 1, $a): T=T_{0}$ at $0<x<H, 0<y<Y_{1} ; T=T_{\mathrm{f}}$ at $0<x<H$, $Y_{1}<y<L$, where $T_{0}$ - initial temperature of "water slug", $T_{\mathrm{f}}$ - initial temperature of gases (the combustion products of forest fuel materials).

Boundary $\left(0<t<t_{\mathrm{d}}\right)$ conditions (Fig. $\left.1, b\right)$ :

$$
\begin{gathered}
x=0, x=H, 0<y<Y_{1} \quad T=T_{f} ; \\
x=0, x=H, Y_{1}<y<Y_{2} \quad \frac{\partial T_{2}}{\partial x}=0 \\
x=0, x=H, Y_{2}<y<L \quad T=T_{\mathrm{f}} ; \\
y=0,0<x<H \quad \frac{\partial^{2} T_{3}}{\partial y^{2}}=0 ; \\
y=Y_{1}, 0<x<H \quad-\lambda_{2} \frac{\partial T_{2}}{\partial y}=-\lambda_{3} \frac{\partial T_{3}}{\partial y}, T_{2}=T_{3} ; \\
y=Y_{2}, 0<x<H \quad-\lambda_{1} \frac{\partial T_{1}}{\partial y}=-\lambda_{2} \frac{\partial T_{2}}{\partial y}-Q_{\mathrm{e}} W_{\mathrm{e}}, T_{1}=T_{2} ; \\
y=L, 0<x<H T=T_{\mathrm{f}},
\end{gathered}
$$

where $Q_{\mathrm{e}}$ - thermal effect of evaporation, $\mathrm{J} / \mathrm{kg} ; W_{\mathrm{e}}$ - mass rate of evaporation, $\mathrm{kg} /\left(\mathrm{m}^{2} \cdot \mathrm{s}\right)$.

A formula was used to calculate the mass rate of evaporation:

$$
W_{\mathrm{e}}=\varphi \rho_{2} V_{\mathrm{d}},
$$

where $\varphi$ - part by volume of liquid in "water slug".

The characteristic longitudinal dimension of "slug" $L_{\mathrm{d}}$ is recalculated similar to [1-3] models for every time step. Transverse size $H_{\mathrm{d}}$ was a constant.

The blowing processes of water vapors from lower $\left(y=Y_{2}, 0<x<H\right)$ boundary and vertical ( $x=0, x=H, Y_{1}<y<Y_{2}$ ) boundaries of "water slug" to the areas parting the water and the flame are not considered in the process of the problem statement. Taking into account these processes, the evaporation takes place less intense (the blowing of vapors significantly decreases the heat flows to the phase transformation surfaces) under other constant conditions. Therefore, the formulated system of Eqs. (1)-(3) and the boundary conditions (4)-(10) describes the heat transfer in the system under consideration (Fig. 1) at as high as possible speeds of water evaporation. All stated below performance evaluations of water evaporation heat using are the high evaluations. The utilization efficiency of extinguishing liquid can be significantly less.

The system of nonstationary partial differential Eqs. (1)-(3) with initial and boundary conditions (4)-(10) was solved by using the methods and algorithms described in [4-7].

\section{Results and discussion}

The numerical investigations were carried out at the following typical values of process parameters [8, 9]: initial water temperature $T_{0}=300 \mathrm{~K}$; the temperature of combustion products of forest fuel 

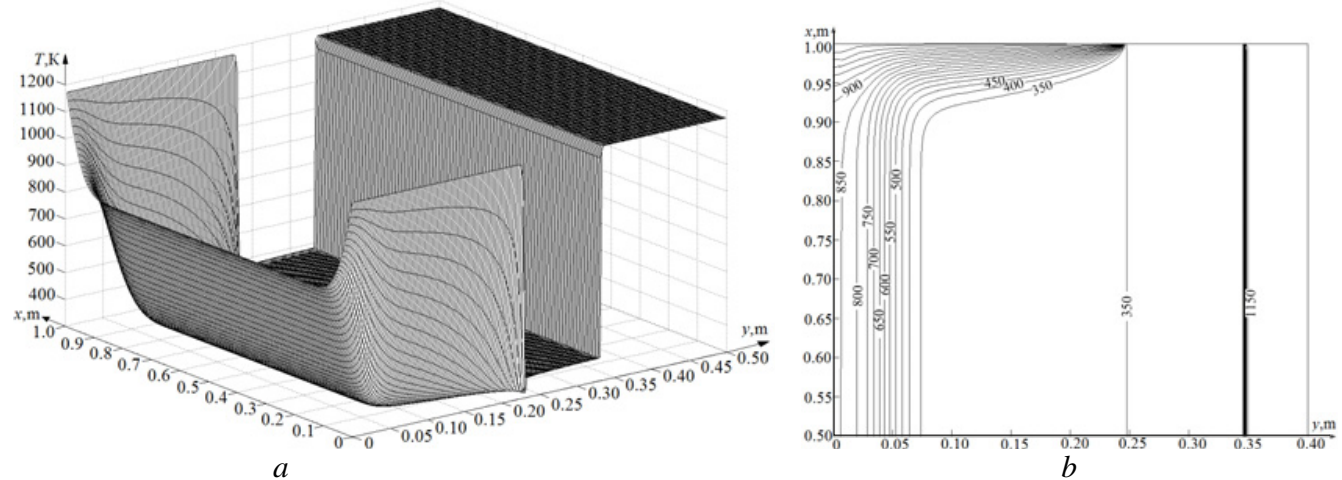

Figure 2. A temperature field $(a)$ and isotherms $(b)$ at $t=0.25 \mathrm{~s}$ and $\varphi=2 \cdot 10^{-4}$.

materials was equal to average fire temperature $T_{\mathrm{f}}=1170 \mathrm{~K}$; thermal effect of water evaporation $Q_{\mathrm{e}}=$ $2.26 \mathrm{MJ} / \mathrm{kg}$; initial "slug" dimensions $H_{\mathrm{d}}=1 \mathrm{~m}, L_{\mathrm{d}}=0.1 \mathrm{~m}$; solution region dimensions $H=1 \mathrm{~m}$, $L=15 \mathrm{~m}$; motion speed of "slug" was a constant from the range of $V_{\mathrm{d}}=0.5 \div 5 \mathrm{~m} / \mathrm{s}$ compared to $[2,3]$. The thermal and physical characteristics of water, water vapors and combustion products of forest fuel materials are presented in $[8,9]$.

Analyzing the macroscopic regularities of "water slug" motion through the high temperature combustion products using the formulated heat transfer model (1)-(11), one of the problems was the determination of values $\varphi$, whereby the temperature of the phase boundary ("liquid - combustion products") ranges within really potential values (not exceeding the steam point at standard atmospheric pressure). Isotherms presented in [2,3] show that the temperature on the boundaries of phase transformation under the motion of single or several water droplets through the high temperature combustion products is approximately equal to $T_{\mathrm{e}}=373 \mathrm{~K}$. The small temperature deviations (up to $5 \mathrm{~K}$ ) on the "liquid - gas" boundary for heat and mass transfer models [2,3] differing from one another can be explained the motion speed change of droplets due to gravity and resistant forces in the sufficiently wide range (from 0.5 to $5 \mathrm{~m} / \mathrm{s}$ ) at $0<t<t_{\mathrm{d}}$ and the various positions of the water vapor blowing boundaries relative to the direction of motion (front, back and side surfaces of droplets). The evaporation conditions of a "slug" moving with a constant velocity $V_{\mathrm{d}}=1 \mathrm{~m} / \mathrm{s}$ were analyzed as a first approximation using the model (Fig. 1) under consideration.

The boundary conditions (9) and the formula for mass rate of evaporation (11) show that the temperature on a "water slug - combustion products" boundary can be close to some constant in time $\left(0<t<t_{\mathrm{d}}\right)$ value $T_{\mathrm{e}}$ under the conditions of $V_{\mathrm{d}}=$ const only at $W_{\mathrm{e}}=$ const $(\varphi=$ const $)$. It was established that the value of part by volume of liquid in the "water slug" fully evaporating under its motion through the flame is $\varphi=0.2 \cdot 10^{-3}$. The temperature of the phase boundary equals to $T_{\mathrm{e}} \approx 375 \mathrm{~K}$ under such conditions and correlates well with results [2, 3]. The evaluation of possible temperature $T_{\mathrm{e}}$ change in the process of "slug" motion at certain $\varphi$ is of interest.

Figures 2 and 3 show the temperature fields and isotherms for the system under consideration (Fig. 1) on the initial $(t=0.25 \mathrm{~s})$ and terminal $(t=10 \mathrm{~s})$ parts of "slug" motion trajectory. Since, the average values of flame height for the forest fires are $10 \div 15 \mathrm{~m}$, hence, the $t \approx 10 \mathrm{~s}$ times at $V_{\mathrm{d}}=1 \mathrm{~m} / \mathrm{s}$ can be considered corresponding to the terminal parts of motion trajectory for the being dropped "slugs". It may be concluded about the fulfillment of the condition $\left(T_{\mathrm{e}} \approx 375 \mathrm{~K}\right.$ at $\left.0<t<t_{\mathrm{d}}\right)$ as follows from the analysis of temperature fields determined for the times characterizing the initial period and the terminal times of "slug" motion.

A found value of part by volume of liquid in a "slug" is $\varphi=0.2 \cdot 10^{-3}$. It shows that the minim of liquid in "water slug" volume is sufficient to decrease the temperature up to as low as practicable values. 

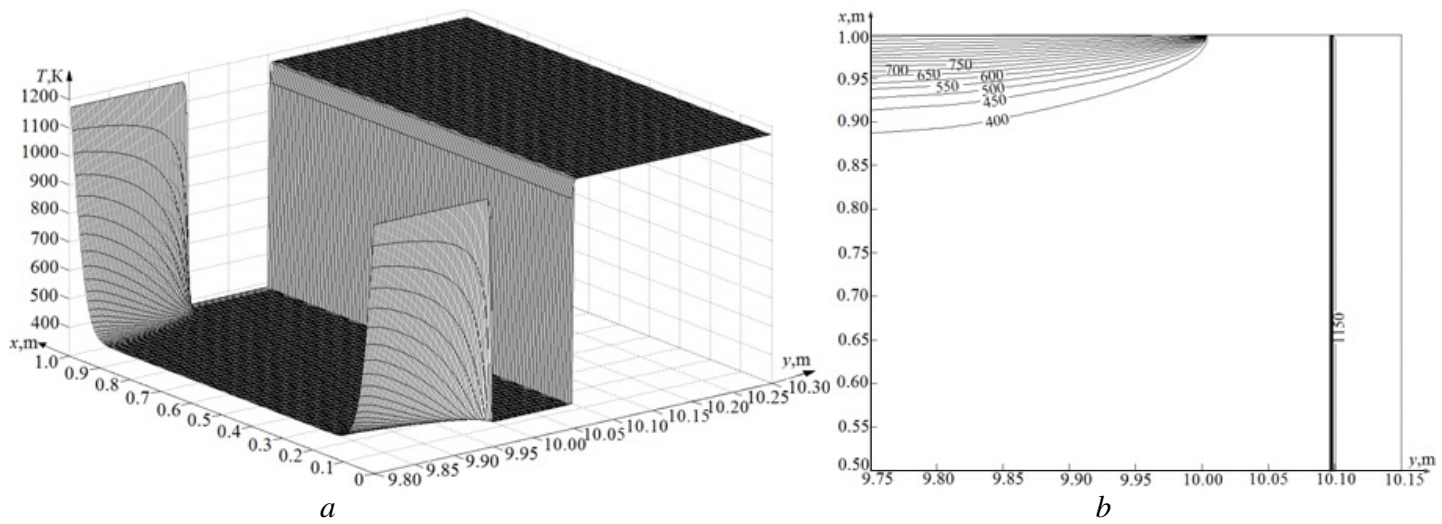

Figure 3. A temperature field $(a)$ and isotherms $(b)$ at $t=10 \mathrm{~s}$ and $\varphi=2 \cdot 10^{-4}$.

It can be explained by the large value of phase transition heat of water ( $Q_{\mathrm{e}}=2.26 \mathrm{MJ} / \mathrm{kg}$ ). Taking into account such heat $Q_{\mathrm{e}}$, the temperature nearby the phase boundary takes up the medium $T_{\mathrm{e}}$ values despite the high temperatures of combustion products $T_{\mathrm{f}}$ (Fig. 3). Since, the temperature of combustion products is slightly varied in accordance with the flame height $\left(T_{1}=T_{\mathrm{f}}\right)$, hence, the evaporation conditions do not change at $0<t<t_{\mathrm{d}}$ (loss in weight of "slug" is not too large and constant).

Figures 2 and 3 show that the low-temperature area is behind the "water slug", hence, the "temperature" track converges. The dynamics of change in characteristic dimensions of this area shows (Figs. 2,3) that the mixture temperature of combustion products and water vapors will close to $T_{\mathrm{f}}$ during the water passing of even a half of way relative to the flame heights under consideration in the initial point of motion trajectory.

It illustrates the low efficiency of water dropping in the combustion area with short times of evaporation.

Isotherms illustrated in Figs. 2, 3 and the temperature fields and the concentration fields of water vapors [2, 3] show that the increasing of the water evaporation area in a "slug" with the use of its atomization is reasonable. The influence of the evaporation process on the temperature in the "slug" motion track is significantly increased under such conditions. Also, the influence of blowing water vapors $[2,3]$ is increased (both the heat transfer with cold water vapors and the substitution by them the oxidizing compound, the gaseous component of fuel and the combustion products will the most important criteria).

The analysis of results concerning a numerical simulation carried out for the heat transfer process in the passing region of "water slug" through the forest fire flame shows that the utilization efficiency of water evaporation heat under typical conditions of extinguishing is less than $0.1 \%$. Taking into account that the estimations carried out are highest, it can be concluded about the expediency of technological advancement of forest fire extinguishing using the aviation (primarily, by using the extinguishing water atomization).

\section{Conclusions}

The new approach concerning the utilization efficiency estimation of water evaporation heat under typical conditions of forest fire extinguishing was proposed. According to the numerical simulation results of heat transfer processes in the passing region of "water slug" through the flame, it can be 
concluded about the low utilization efficiency of extinguishing liquid within a framework of modern technologies of forest fire extinguishing using the aviation.

The investigation was performed by Russian Science Foundation grant (project 14-39-00003).

\section{References}

[1] O.V. Vysokomornaya, G.V. Kuznetsov, and P.A. Strizhak, Journ. of Eng. Phys. and Thermophys. 1, $86(2013)$

[2] D.O. Glushkov, G.V. Kuznetsov, and P.A. Strizhak, Math. Probl. in Eng. 2014 (2014)

[3] P.A. Strizhak, Journ. of Eng. Phys. and Thermophys. 4, 86 (2013)

[4] D.O. Glushkov, G.V. Kuznetsov, and P.A. Strizhak, Russ. Journ. of Physic. Chem. B 5 (2014)

[5] D.O. Glushkov, G.V. Kuznetsov, and P.A. Strizhak, Adv. in Mech. Eng. 2014 (2014)

[6] O.V. Vysokomornaya, G.V. Kuznetsov, and P. A. Strizhak, Math. Probl. in Eng. 2014 (2014)

[7] P.A. Strizhak, Journ. of Eng. Thermophys. 4, 20 (2011)

[8] J.O. Hirschfelder, C.F. Curtiss, and R.B. Bird, Molecular Theory of Gases and Liquids (John Wiley and Sons, New York, 1954)

[9] N.B. Vargaftik, L.P. Filipov, A.A. Tarzimanov, E.E. Totskii, Handbook of Thermal Conductivity of Liquids and Gases (CRC Press, Inc., Boca Raton, 1994) 